\title{
Fluxgate Magnetometer with an Orthogonal Flux Guide
}

\author{
Maha Aldoumani \\ Cardiff University \\ School of Engineering
}

\author{
Noor Aldoumani \\ Cardiff University \\ School of Engineering
}

\begin{abstract}
This paper will examine the developed ANSYS model to test advanced materials, i.e. amorphous and metaglas, as well as to optimise the geometry of the fluxgate magnetometer. In other words, the current chapter presents an optimised study of the materials and geometry of the magnetometer which provides savings in terms of material usage as well as the employed electric current to produce an equivalent magnetic field. This design has been developed using the PCB wherein a magnetometer consisting of a planar fluxgate structure with an orthogonal ferromagnetic fluxguide has centrally been situated over the magnetic core.
\end{abstract}

\section{Keywords}

Amorphous and metaglas materials, Fluxgate magnetometer, Flux guide, geometry of the magnetometer.

\section{INTRODUCTION}

Traditional fluxgate magnetic sensors typically possess high device sensitivity, low noise density and excellent sensing accuracy. However, they are still inferior to other magnetic sensors in the aspects of bulky volume of coils, higher power consumption and lower integration capability [1]. Other competitive magnetometers such as anisotropicmagnetoresistance (AMR), tunneling magnetoresistance (TMR), giant-magnetoresistance (GMR), magnetoimpedence (MI) and magnetotransitor (MT) devices have been extensively studied and reported [2]. Nonetheless, with the progress of system miniaturization, the recent advance of miniature fluxgate sensors using CMOS technologies has been promising. Fluxgate magnetometers are typically applied in craft navigation, military detection and medical recognition [3]. So far prospective applications of micro-fluxgates have been considerably developed for modern digital navigation , thoracoscopic surgery, and nondestructive inspection [4]. On device characterization of fluxgate magnetometers, several earlier works concluded that the sensing methodology and sensitivity enhancement by adopting the multiple harmonic frequency analysis techniques are practicable [5]. In addition to magnetic core materials, design of excitation and pick-up coils is also critical to determine the functioning of a fluxgate device. Designs for planar excitation and pick-up coils or three-dimensional (3-D) coils were previously investigated [67]. Traditional fluxgate magnetic sensors typically possess high device sensitivity, low noise density and excellent sensing accuracy. The traditional coils of fluxgate sensors, commonly wire-wound, are usually characterized as high sensitivity and low noise; but, they are still too bulky and system-incompatible to meet the dimensional requirements of a miniature product. This paper will discuss the evolution and the results of the model sensor that has been designed by ANSYS software, this work allows to define a design way for the development of the magnetic fluxgate sensor, illustrate the results of the model and compare the output with many cases that has been made such as evaluate the excitation current value that ensures the saturation for ferromagnetic material and how the current is affecting the $\mathrm{BH}$ curve which in turn will affect the sensitivity of the sensor, different number of turns and analyse the $\mathrm{BH}$ curves of each case, Compare the output voltage with different thickness ferromagnetic materials, different wire width, and different core width.

The advantage of using the PCB technology is to reduce the cost and the simplicity of prototyping. The magnetic fluxgate sensor that has been modelled is an amorphous metal that is commercially available and which is neither electroplated nor sputter produced as it is the case with most ferromagnetic materials. The materials involved in the sensor include $25 \mu \mathrm{m}$ for Vitrovac 6025-X, the $20 \mu \mathrm{m}$ for Vitrovac $6025-\mathrm{Z}$ and the $16 \mu \mathrm{m}$ for Metglass 2714-A). In this way it will be easily to optimise, build and analyse the design then get the results. The first step in the work is to find a suitable geometry for the model which will be optimised utilising ANSYS software to develop and analyse the sensor.

\section{BASIC SENSOR STRUCTURE}

The architecture of the design has been developed using the PCB and a comparison between the various cases has been carried out in the current work. The first stage that has been taken into consideration is the field distribution within the core by looking at the flux line diagram through the core. This structure consists of a cross shaped ferromagnetic core, a planar excitation coil and four sensing coils. The four sensing coils and the excitation coil are placed on two different metal layers in a multilayer PCB structure. The ferromagnetic cores are placed in a cruciate shape on the excitation coils diagonally, ( Figure 1) and (Figure 2). Then the excited field will be in opposite directions in pairs, so they will cancel each other in the sensing coils. The sensing coils are placed which are underneath the excitation coil and centred on the four endpoints of the cross shape core. Both orthogonally cylindrical ferromagnetic tube (which was placed at the top of the core) and set of ferromagnetic cubes (which was placed underneath the core) are acting as a fluxguide in order to concentrate and guide magnetic fields. So the tube will deflect the field to be passed through the core, then the ferromagnetic cubes will emit the field into the sensing coils.

The ferromagnetic material that has been used for the core is an amorphous alloy known under the name of Vitrovac 6025 (6025-Z with a thickness of $20 \mu \mathrm{m}$ and $6025-\mathrm{X}$, with a thickness of $25 \mu \mathrm{m})$ of a high relative permeability $(\mu \mathrm{r} \cong$ $105)$. Also, the value of BS of this material is $0.55 \mathrm{~T}$. The Vitrovac alloys (6025-Z and 6025-X) can be recognised from the shape of their hysteresis loop, this property affects the excitation current that is needed to ensure the saturation of the ferromagnetic material.

In the fluxgate sensor that has been modelled, the following parameters are modelled by simulation: Excitation metal layers thickness: $25 \mu \mathrm{m}$, Sensing metal layer thickness: 20 $\mu \mathrm{m}$, Metal lines pitch: $400 \mu \mathrm{m}$,Vitrovac thickness: $25 \mu \mathrm{m}$ $(6025 \mathrm{X})$ and $20 \mu \mathrm{m}(6025 \mathrm{Z})$ 
In addition to the fixed basic parameters of the design and the size of the sensor, there are some parameters that have to be optimized such as the number of turns of the excitation coil and the number of turns of the sensing coils. The four sensing coils are placed in separate layers of the excitation coil. In order to achieve a better sensitivity, a large ferromagnetic material which requires a larger current is required. The dimensions were finally set to be $40.0 \times 3.5 \times 0.025 \mathrm{~mm}$ (for the Vitrovac 6025-X). A cylindrical ferromagnetic tube was placed on the top of the centre of the crossed-core fluxgate and these acts as the fluxguide in order to concentrate the magnetic fields then emit the fields into the planar cruciform core.

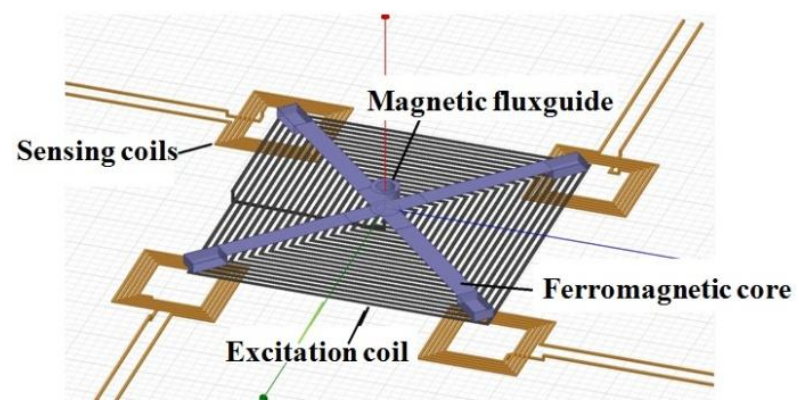

Fig. 1: schematic of the 3-axis planar fluxgate magnetic sensor realized.

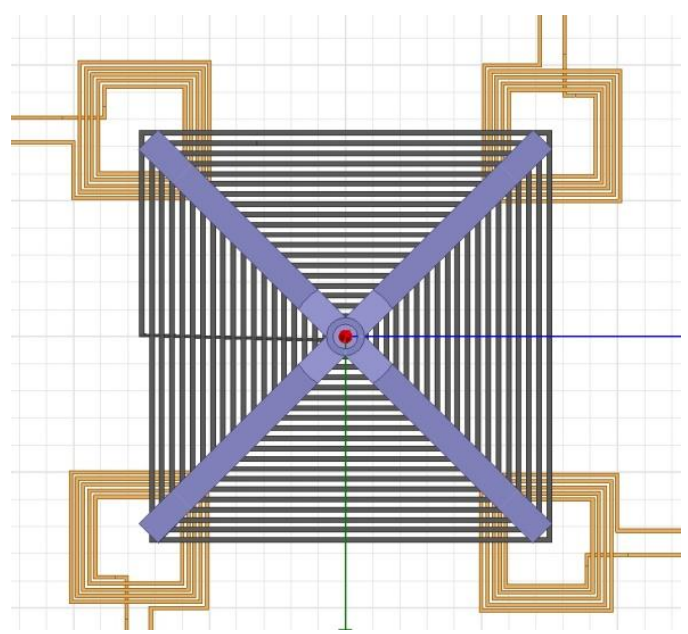

Fig. 2: view of the 3-axis planar fluxgate magnetic sensor.

\section{THE WPRKING PRINCIBLE OF THE SENSING DEVICE}

The system has been investigated while studying the effect of the fluxguide on the performance of the device. In general, the working principle of the planar fluxgate including the cruciform core alongside a vertically mounted fluxguide has been studied. In such a system, the magnetic field in the $\mathrm{X}$ axis or Y-axis detections is distributed as flux lines which are comparable with those of the planar device in particular nearby the ends of the core as shown in (Figure 3 a) and (Figure 4 a). However, the interior part of the fluxguide system is different and this results in a trivial effect on both ends of the core as illustrated in (Figure 4 a).

Through the simulation of flux line distribution, it is confirmed that the tri-axis sensor with a fluxguide does not generate significant interference for $\mathrm{X}$-axis (or $\mathrm{Y}$-axis) detection and surely obtain the vector component of magnetic fields in $\mathrm{X}$-axis (or Y-axis) as the planar one. On the other hand, the measurement of the field in the Z-direction will involve that the magnetic field lines will be parallel to the longitudinal orientation of the fluxguide. This is evidently demonstrated (Figure $4 \mathrm{~b}$ ) which reveals that the fluxlines near the top of the fluxguide are slightly bent and hence guided into its interior and then transferred along the $\mathrm{X}$ direction or Y-direction and eventually diverged into air (or vacuum).

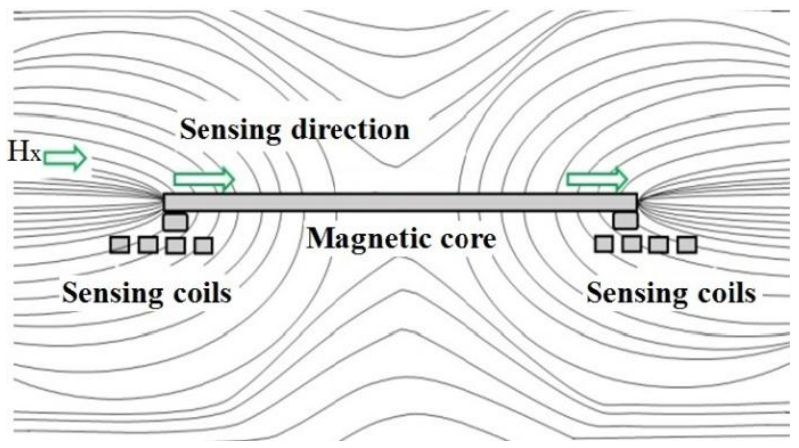

Fig. 3 a): The fluxlines of the sensing principle of the fluxgate in $\mathrm{X}$ axis direction without fluxguide.

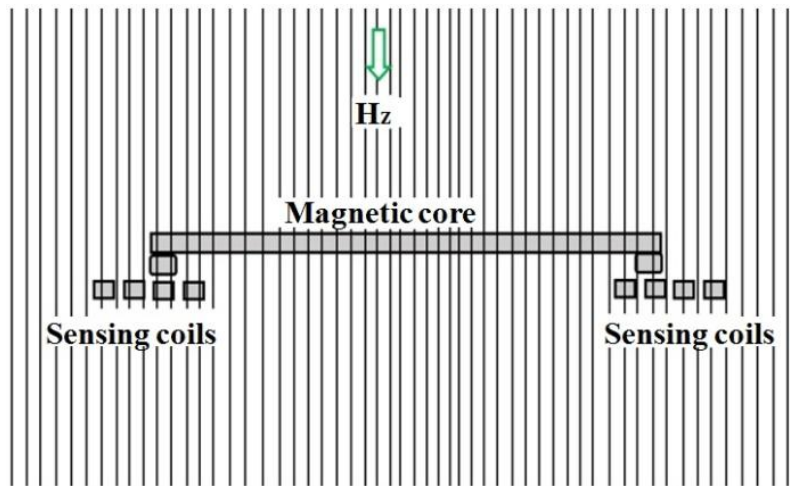

Fig. $3 \mathrm{~b}$ ): The fluxlines of the sensing principle of the fluxgate in $\mathrm{Z}$ axis direction without fluxguide.

It can be clearly seen that the magnetic fluxlines gather at the ends of the core, in addition to that these fluxlines are compressed into the core until they spread out to the air again at the other end of the core. This phenomenon creates a flux density around the end points of the core in the sensor. In this case, the flux density that has been generated will be converted to voltage induced through the two pick-up coils. It can be conclude that orthogonally cylindrical ferromagnetic tube and set of ferromagnetic are acting as a fluxguide in order to concentrate and guide magnetic fields. So the tube will deflect the field to be passed through the core, then the ferromagnetic cubes will emit the field into the sensing coils. 


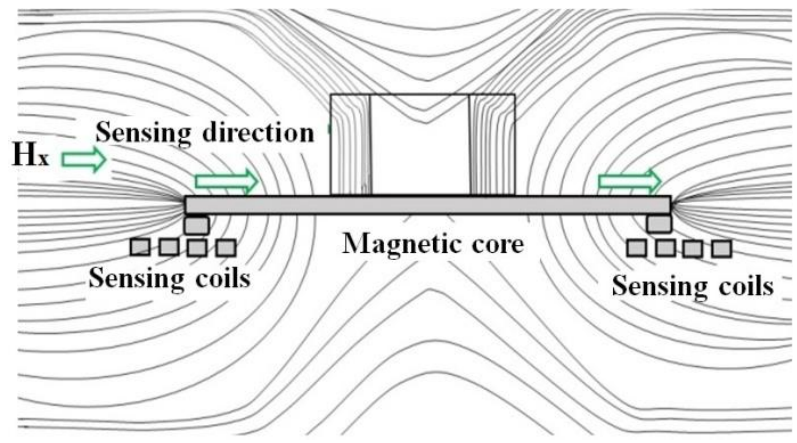

Fig. 4 a): fluxlines of the sensing principle of the fluxgate with the fluxguide in $X$ axis.

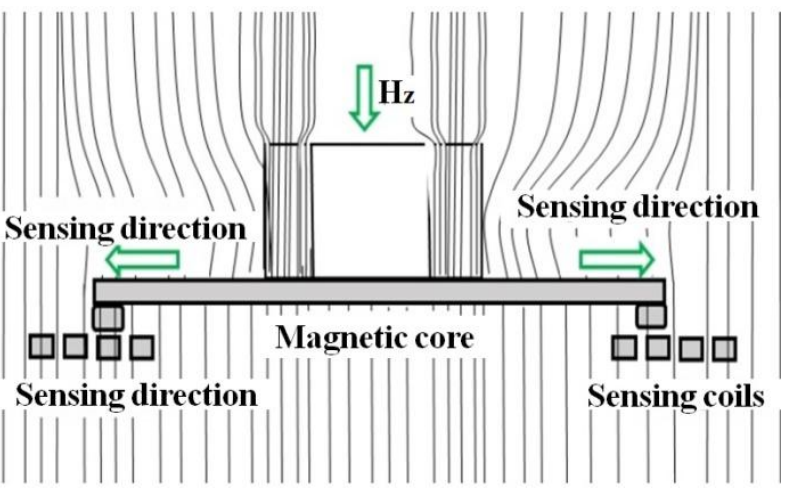

Fig. 4 b): fluxlines of the sensing principle of the fluxgate with the fluxguide $Z$ axis.

\section{THE SENSOR DEVLOPMENT AND SIMULATION RESULTS}

It is important to find the optimal dimensions of the proposed fluxgate in terms of the geometry of the core, geometry of the excitation and sensing coils in addition to the number of turns alongside the width of the wire of the excitation coil. The simulation of the fluxgate sensor has taken place using the Maxwell Ansys software based on finite element analysis. Several simulations were performed to find the best geometrical configuration of the ferromagnetic core. After an iterative process of design optimisation, the ferromagnetic material shape selected was a pair of rectangular sheets with dimensions of $40.0 \times 3.5 \times 0.025 \mathrm{~mm}$ (for Vitrovac 6025-X). The main issue encountered in this iterative optimisation process is the creation of a good mesh for the model of the structure.

The design stages involved in the current project started with finding the amount of the excitation current value that ensures that the ferromagnetic material is saturated. This has been achieved using magnetostatic simulations to evaluate the excitation peak current necessary to saturate the ferromagnetic material.

\subsection{The influence of the induced current on the core material}

The first step in the analysis is to determine the value of the excitation current that saturates the ferromagnetic material. This has been done using current values between $450 \mathrm{~mA}$ and $750 \mathrm{~mA}$. The effect of the induced current on the magnetic properties of the ferromagnetic core material has been analysed. As illustrated in (figure 5) it can be determine that all curves have different output voltages and hence are not all of them are suitable to saturate the core that will be used for a fluxgate magnetometer.
Furthermore the different shapes of the curves that has been obtained from the different amount of currents values indicating that the amount of $680 \mathrm{~mA}$ was sufficient to cause saturation of the core of the fluxgate and perform as a good fluxgate sensor. However, it has been found that some of these amounts of the currents that has been applied do not saturate the core to make a good fluxgate at all. It is possible to grade all the amount of the currents that has been applied among themselves and conclude that the amount of $(680 \mathrm{~mA})$ is the most suitable value to saturate the ferromagnetic material that has been used for the core, the amount of $(750 \mathrm{~mA})$ is an average to saturate the material and the amount of $(550 \mathrm{~mA})$ and $(450 \mathrm{~mA})$ are the least suitable amount of currents to saturate the ferromagnetic material to be used as cores in magnetometers. It has been observed that when the current was either high $(750 \mathrm{~mA})$ or low $(450 \mathrm{~mA})$, the material has not been saturated. However, at a value inbetween those, i.e. $680 \mathrm{~mA}$, the material has reached the saturation point which is desirable. It has also been observed that for the geometrical configuration utilised in the current study, the optimum amount of $680 \mathrm{~mA}$ was sufficient to cause saturation and get the highest sensitivity. This is in contrast to the case with the low and high currents which caused the sensitivity of the core to be dramatically dropped.

In the case of Vitrovac $6025-\mathrm{X}$, a current of $680 \mathrm{~mA}$ has been observed to cause saturation of the ferromagnetic material, (Figure 6), with Bmax around 0.54 $\mathrm{T}$. The distribution of the magnetic flux density (B) along the core while the core excitation is realised has been simulated and evaluated by ANSYS Maxwell.

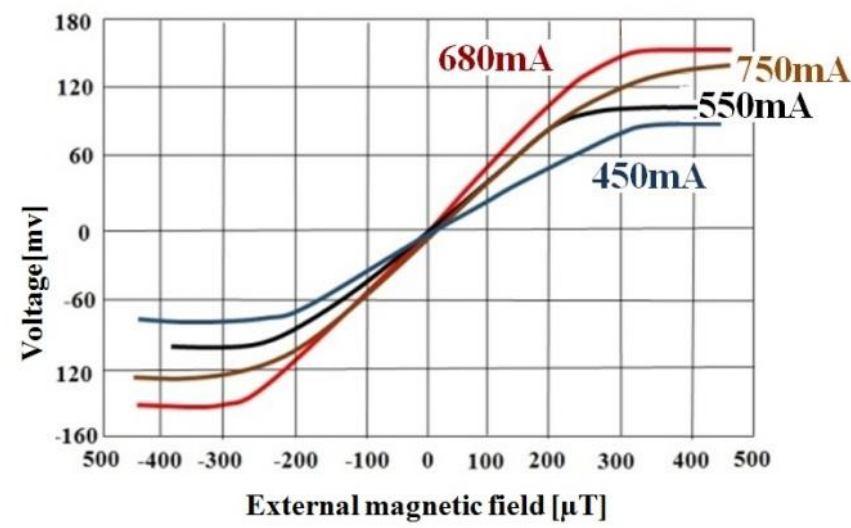

Fig. 5: The effect of the induced current on the magnetic properties of the ferromagnetic core material.

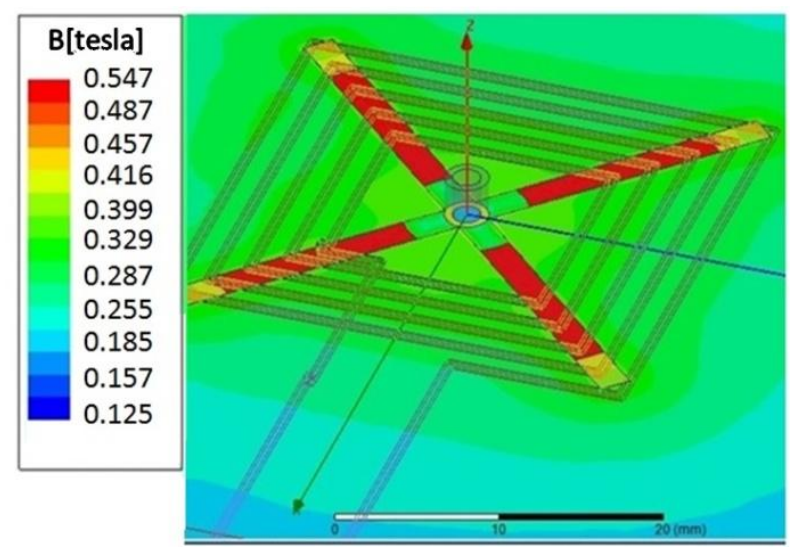


Fig. 6 :The determination of the saturation point of the ferromagnetic core material.

In order to select the suitable current to saturate the ferromagnetic material to be used as a core in magnetometers, a selective parameter approach has been proposed which shows the sensitivity of the sensor. A different sensitivity values have been obtained for the various excitation currents that have been applied, and these are summarised in Table 1 it can be evidently seen that the highest sensitivity is observed at a current of $680 \mathrm{~mA}$. In Table 1 clearly differentiates between a high sensitivity of the sensor and a low sensitivity of the sensor. Furthermore, it is possible to classify values of the sensitivity of the different currents values that has been applied among themselves and conclude that in the amount of $(680 \mathrm{~mA})$ can get the highest sensitivity, in the amount of $(550 \mathrm{~mA})$ can get an average sensitivity and the amount of $(450 \mathrm{~mA}$ and $750 \mathrm{~mA})$ can get the least sensitivity of the sensor.

Table 1: The various excitation current values with sensitivity in the range

\begin{tabular}{cc}
\hline $\begin{array}{c}\text { The various excitation } \\
\text { current values }[\mathrm{mA}]\end{array}$ & $\begin{array}{c}\text { Sensitivity of the } \\
\text { sensor[mV/ } \mu \mathrm{T}]\end{array}$ \\
\hline 450 & 0.35 \\
550 & 0.41 \\
680 & 0.48 \\
750 & 0.38 \\
\hline
\end{tabular}

From the simulation exercises, it has been observed that the middle section of the core has been saturated with significantly larger amounts when compared to the ends of the core, (Figure 7). This is evident from the larger amount of the magnetic flux observed in the middle of the core material.

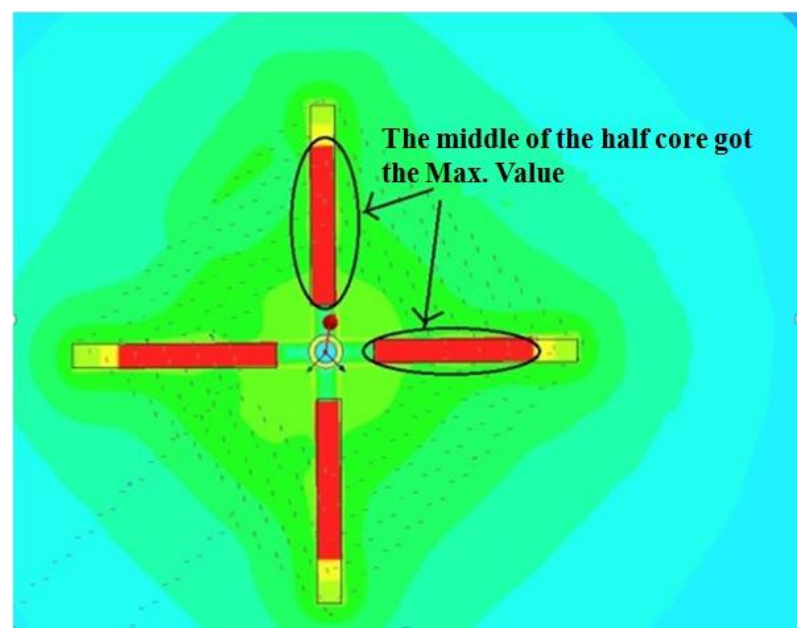

Fig. 7: The saturation process of the ferromagnetic core material at $680 \mathrm{~mA}$.

\subsection{The Influence of the number of turns on the Coil}

In order to select the suitable coil to be used in magnetometers, the same model and design but with different number of turns (10,20 and 40 turns). As illustrated in (Figure 8) it can be established that each curve has a different output voltages were obtained depending on the applied number of turns. Hence are not all of them are suitable to be used as magnetic core for a fluxgate magnetometer. Moreover, the different shapes indicating that in the case of (40 turns) should perform as a good fluxgate sensor.

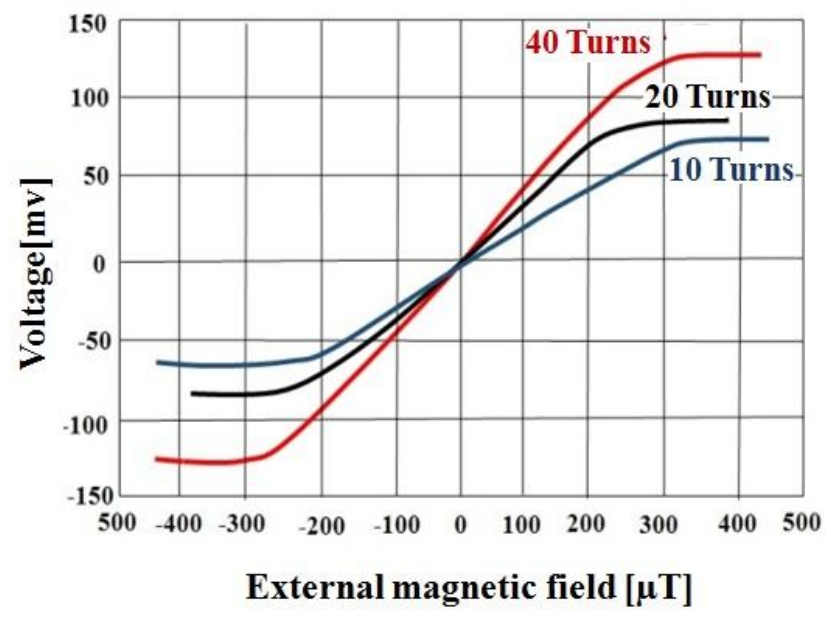

Fig. 8 : The effect of the number of the turns on the magnetic properties of the ferromagnetic core material.

In addition to that, it has been found that some of these cores do not make a good fluxgate at all. It is possible to grade all the curves that has been obtained among themselves and conclude that in the case of (40 turns) is the most suitable coil for the fluxgate, in the case of (20 turns) is an average coil and the case of (10 turns)is the least suitable coil to be used in magnetometers. However, the higher the number is, the better the suitability of that coil as a fluxgate sensor. The increase in the number of turns will result in an increase in the size of the excitation coil and hence an increase in the output voltage, (Figure 9).

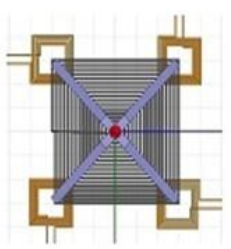

(a)

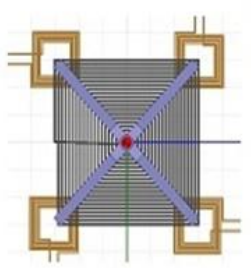

(b)

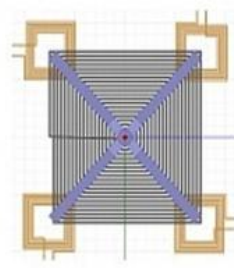

(c)
Fig. 9: The change in number of turns of the excitation coil: (a) 10 turns, (b) 20 turns and, (c) 40 turns.

The reduction in the number of turns of the excitation coil gives a lower output voltage value, therefore; the maximum voltage can be obtained with the maximum possible number of turns, i.e. in this case 40 turns, of the employed model (see Figure 10). Approximate finite element method simulation of 3-dimensional as used in this study in order to show the saturation of the core material when the maximum number of turns, i.e. 40 turns, is used. Magnetic field strength's variation is colour-coded: the highest intensity being denoted by red, 
the lower by yellow and lowest by green. The scale of the magnetic field strength in this simulation is arbitrary.

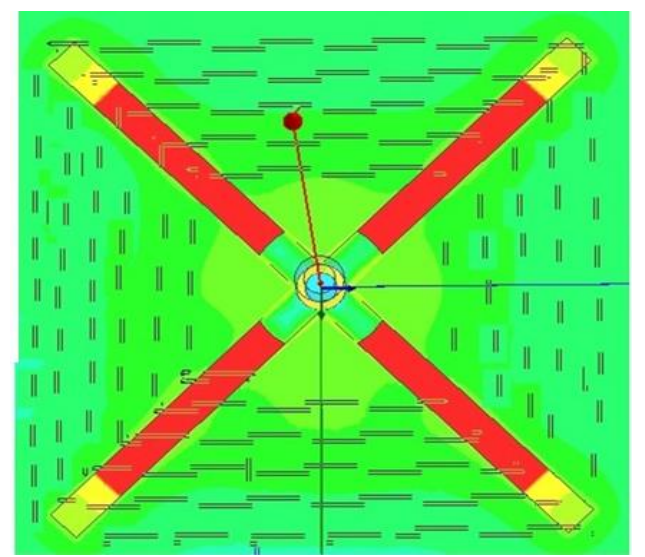

Fig. 10: The saturation of the core material when the maximum number of turns, i.e. 40 turns, is used.

\subsection{The Influence of the Ferromagnetic material on the Hysteresis Loop}

To characterize the most suitable core for the fluxgate, the same model design has been used but with different ferromagnetic, Unlike the previous simulations of the Vitrovac $6025-\mathrm{X}$ which was saturated at $680 \mathrm{~mA}$, a new configuration of the same material called Vitrovac $6025-\mathrm{Z}$ of a smaller thickness has been employed and investigated. It is worthwhile mentioning that the thickness of the Vitrovac $6025-\mathrm{X}$ is $25 \mu \mathrm{m}$ whereas that of the Vitrovac $6025-\mathrm{Z}$ is $20 \mu \mathrm{m}$. As illustrated in (Figure 11) that it can be established that the three different materials that has been used have different hysteresis loop due to the difference thickness of that materials and hence are not all of them are suitable to be used as magnetic core for a fluxgate magnetometer. Furthermore the different shapes of the B-H loops of the materials indicating that the smaller the thickness of the core, the lower the amount of the used current, i.e. less power consumption, the lower the linearity error and the higher the sensitivity of the device which should perform as a good core for the fluxgate sensor. However, it has been found that some of these materials do not suitable for a good core fluxgate at all. It has been found from (Figure 11) that interestingly, when an additional material, i.e. Metglas2714A, has been investigated in terms of the influence of the core thickness on the output voltage. From (Figure 11) it is possible to grade all the materials among themselves and conclude that the Metglas $2714 \mathrm{~A}$ of thickness $16 \mu \mathrm{m}$ provides very close results in terms of the saturation current to those obtained by $6025-\mathrm{Z}$ with the added advantage of a smaller thickness of the core. However, the sensitivity of the Metglas 2714A is significantly lower along with a higher linearity error when compared to the 6025-Z.

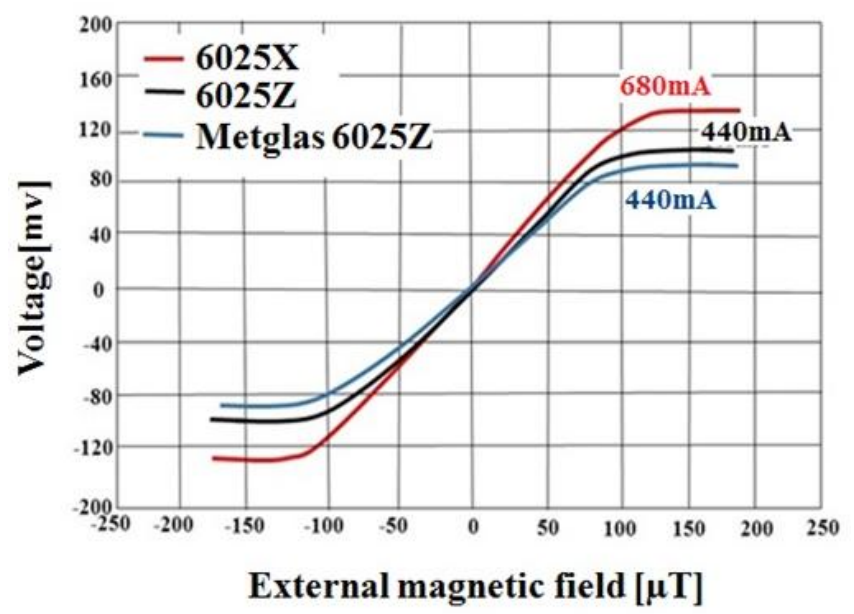

Fig. 11: The influence of the core thickness on the output voltage of the device.

\subsection{The Influence of the Ferromagnetic Wire Width on the Hysteresis Loop}

The different magnetic flux density (B) has been measured through the core length in terms of the variation of the wire width with an excitation current of $680 \mathrm{~mA}$. Looking at all data in (Figure 12) shows the different wire width from $(0.1 \mathrm{~mm}-0.5 \mathrm{~mm})$, it can be established that all cases of different wire width have a different magnetic flux density (B) and hence are not all of them are suitable to be used as magnetic core for a fluxgate magnetometer. As shown in (Figure 12). It can be seen that the flux density B will be the highest value in the case of $(0.1 \mathrm{~mm})$ wire width which is suitable to be used as core for the fluxgate magnetometer. Furthermore the different magnetic flux density (B) that has been obtained indicating that in the case of $(0.1 \mathrm{~mm})$ wire width should perform as a good fluxgate sensor. However, it has been found that some of these cores do not make a good fluxgate at all. it is possible to grade all cases of the difference of the wire width among themselves and conclude that in the case of $(0.1 \mathrm{~mm})$ is most suitable materials to be used as a core of the fluxgate magnetometer, In the case of $(0.2 \mathrm{~mm}$ and $0.3 \mathrm{~mm})$ is an average material and the case of $(0.4 \mathrm{~mm}$ and $0.5 \mathrm{~mm}$ ) are the least suitable materials to be used as cores in magnetometers. It can be conclude that magnetic flux density (B) is inversely proportional to the wire width, i.e. the smaller the wire width the higher the flux density which is desirable.

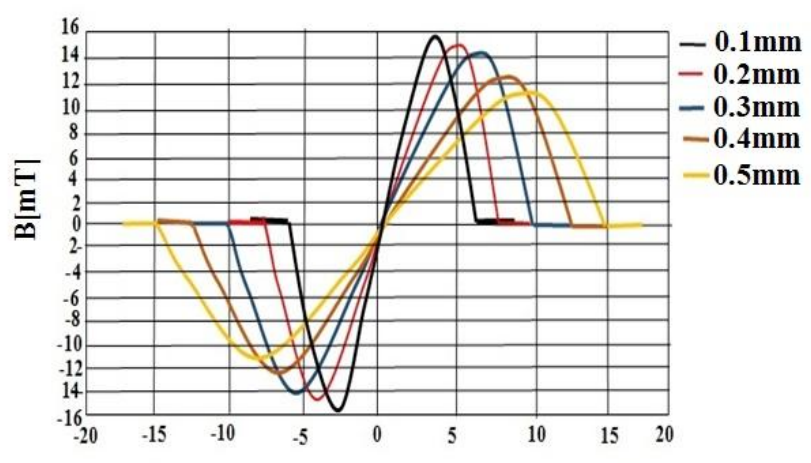

The distance from the core junction(mm)

Fig. 12: The influence of the wire width on the flux density through the core. 


\subsection{The Influence of the Ferromagnetic Core Width on the Hysteresis Loop}

When the same current of $680 \mathrm{~mA}$ was applied with the same modelled design, the core width has been investigated in terms of flux density and saturation of the core. Furthermore the different magnetic flux density (B) has been measured through the core length in terms of the variation of the core width. Looking at all curves in (Figure 13) shows the different core width from $(2 \mathrm{~mm}-5 \mathrm{~mm})$, it can be established that all cases of different core width have a different magnetic flux density (B) and hence are not all of them are suitable to be used as magnetic core for a fluxgate magnetometer. As shown in (Figure 13), it can be seen that the flux density B will be the highest value in the case of $(2 \mathrm{~mm})$ wire width which is suitable to be used as core for the fluxgate magnetometer. Furthermore the different magnetic flux density (B) that has been obtained indicating that in the case of $(2 \mathrm{~mm})$ core width should perform as a good fluxgate sensor. However, it has been found that some of these cores do not make a good fluxgate at all. it is possible to grade all cases of the difference of the core width among themselves and conclude that in the case of $(2 \mathrm{~mm})$ is most suitable materials to be used as a core of the fluxgate magnetometer, in the case of $(3 \mathrm{~mm})$ is an average material and the case of $(4 \mathrm{~mm}$ and $5 \mathrm{~mm})$ are the least suitable materials to be used as cores in magnetometers. It can be conclude that magnetic flux density (B) is inversely proportional to the core width, i.e. the smaller the core width the higher the flux density which is desirable.

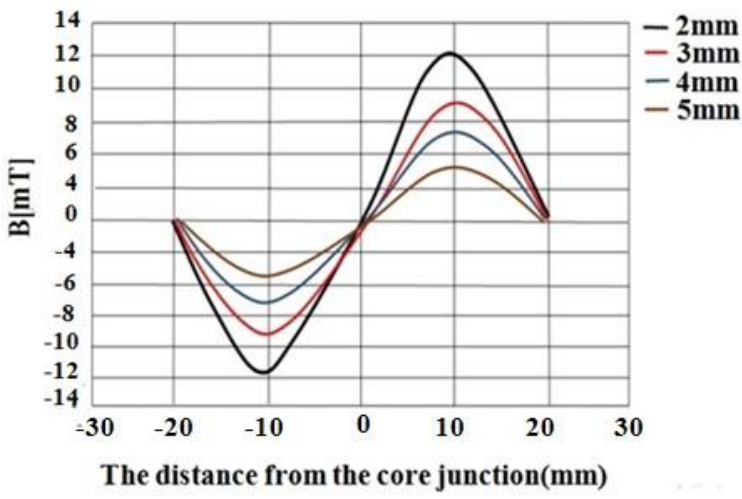

Fig. 13: The influence of the core width on the flux density through the core.

\section{CONCLUSION}

The work presented in this paper provides a novel fluxgate design that has ability to concentrate and guide the field in a highly efficient manner by employing orthogonally cylindrical ferromagnetic tube and set of ferromagnetic cubes in order to deflect and emit the field in the desired path. A micro-fluxgate sensor has been developed during the current study utilising ANSYS to optimise the geometry of the fluxgate magnetometer. In other words, an optimised study of the geometry of the magnetometer provides savings in terms of material [8] usage as well as the employed electric current to produce an equivalent magnetic field. The magnetic fluxgate sensor that has been modelled is made of an amorphous metal $(25 \mu \mathrm{m}$ for Vitrovac $6025-\mathrm{X}$, the 20 $\mu \mathrm{m}$ for Vitrovac $6025-\mathrm{Z}$ and the $16 \mu \mathrm{m}$ for Metaglass 2714-A) that is commercially available. The optimisation exercise has been carried out by changing the current level in the fluxgate where the optimum value of the current level that caused saturation of the core material as well as provided the highest possible sensitivity was found to be around $680 \mathrm{~mA}$. Also, the optimisation process included optimising the number of turns where it has been found that the optimum value of turns should be around 40 turns. The wire width of the coil has also been investigated using ANSYS where the optimum width of the wire was found to be between 0.1 and $0.5 \mathrm{~mm}$. It was found that the flux density $B$ is inversely proportional to the wire width, i.e. the smaller the width the higher the flux density which is desirable. It was also found that the smaller the width of the core the higher the flux density and therefore the faster the saturation of the core material.

\section{ACKNOWLEDGMENTS}

Sincere thanks to Dr. Anoor for his scientific guidance and for participating in discussions about this project.

7. REFERENCES

[1] Marshall S V, "An Analytic Model for the Fluxgate Magnetometer”, IEEE Trans. Magn.1967.(3):287-289.

[2] Ripka P, "New directions in fluxgate sensors," Journal of Magnetism and Magnetic Maerials 2000.(215):pp.735739 .

[3] Dolabdjian C, Saez S, Toledo A R , Robbes D "Signalto-noise improvement of bio-magnetic signals using a flux-gate probe and real time signal processing". Rev. Sci. Instrum 1998.(69):pp. 3678-3680.

[4] Tseng J Z, Wu C C, Dai C L "Modeling and manufacturing of a micromachined magnetic sensor using the CMOS process without any post-process". Sensors 2014. (14):pp. 6722-6733.

[5] Ripka P "Advances in fluxgate sensors. Sens. Actuators Phys. 2003.(106):pp. 8-14.

[6] Shimada J, Itoh K, Yamaguchi S, Nishikawa A, Miyazaki F, "Magnetic Navigation System for Thoracoscopic Surgery of the Pig Lung Partial Resection with Transbronchial Marking". Int. Congr. Ser. 2005.(1281):pp.752-755.

[7] Munschy M, Boulanger D, Ulrich P, Bouiflane $M$. "Magnetic mapping for the detection and characterization of UXO: Use of multi-sensor fluxgate 3-axis magnetometers and methods of interpretation". J. Appl. Geophys. 2007.(61):pp.168-183. 